\title{
Discrete Networked Dynamic Systems with Eigen-Spectrum Gap: Analysis and Performance
}

\author{
Magdi S. Mahmoud $^{1 *}$ and Bilal J. Karaki ${ }^{1}$ \\ ${ }^{1}$ Systems Engineering Department, KFUPM, Dhahran 31261, Saudi Arabia \\ *Corresponding author
}

\begin{abstract}
Article Info
Keywords: Discrete network dynamic systems (DNDS), Mode separation, Reduced-order modelling, State dependent networks

2010 AMS: 15A39, 68R10, 93A15, 93B52, 93D05, 94C15

Received: 24 October 2020

Accepted: 26 February 2021

Available online: 05 March 2021
\end{abstract}

\begin{abstract}
This paper provides a detailed analysis and performance treatment of a class of discretetime systems with an eigen-spectrum gap coupled over networks. We deploy tools from time-scale modeling (TSM) theory to develop rigorous reduced-order models to aid in the stability analysis of these multiple time-scale networked systems over fixed and undirected graph topology. We establish that the controller gain matrices can be determined by solving convex optimization problems in terms of finite linear matrix inequalities with prescribed $\mathbb{H}_{\infty}$ and $\mathbb{H}_{2}$ performance criteria. As demonstrated by simulation studies, the ensuing results provide designers with a network-centric approach to improve the performance and stability of such coupled systems.
\end{abstract}

\section{Introduction}

The usefulness of time-scale modeling (TSM) theory for the analysis and synthesis of dynamical control systems with slow and fast dynamics has been broadly recognized as a strong technique for over four decades [1, 2]. Different control methodologies have received great attention of various researchers in the theory of control systems that comprises time-scales [3]-[6]. An important feature of the existing results is that the control analysis and synthesis are accomplished in two stages, such that a suitable reduced-order dynamics is treated at each stage. Order reduction and control has been extended to discrete systems with two time scale [7]-[9] based on explicit invertible-transformations where quasi-steady-state is assumed [10]-[13]. It has been demonstrated that the discrete time dynamics can be reduced to (a) a slow sub-dynamics with large eigenvalues near the the unit disk and (b) a fast sub-dynamics with eigenvalues distributed near the origin of the disk. This decomposition can be satisfied if an inequality relating the norms of subsystem matrices holds. Therefore, this structure allows the user to implement feedback control using different gain matrices. Along with the enormous advancement of control theory, technological development of controlling a group of agents has been widely investigated and received increasing demands. A common structure for controlling a group of agents is the distributed cooperative and coordinated control techniques [14]-[19]. Recently, distributed coordination of multi-agent systems have received a tremendous interests in a wide range of practical applications, mainly including engineering, ecology, biology and sociology [20]-[30].

On another research avenue, discrete networked dynamic systems (DNDS) provides a high-level treatment of a general class of linear discrete-time dynamic systems interconnected over an information network processed in discrete-time environment, exchanging relative state measurement or output measurements. It seems encouraging that by exploiting the impact of the network properties, additional features of the dynamical systems can be revealed [31]. On a parallel development in view of the available results, it turns out that research avenues in multiagent systems offer great opportunities for further developments from theoretical, simulation and implementations standpoints [32, 33]. TSM theory [34, 35] is attractive for establishing these approximations as the obtained reduced-order dynamics guarantees the asymptotic behavior of the coupled-consensus 
dynamics and give a viable estimate of the performance of the network trajectories through a simpler set of equations, compared to the original complex structure. Reduced-order modeling and synchronization of a network of homogeneous linear agents that comprises two time-scale behavior over fixed and undirected graph topology are investigated in [36]. However, most of the existing results do not employ the time-scale separation that normally appears between the agent-layer and the network-layer. By adopting advantage of this framework, the technique addressed in this article is, therefore, flexible to be applied to a broad range of agent models and wide range network controllers. In addition, it gives important insights into the interplay between design parameters such as controller parameters and communication topology on the behavior of coupled-consensus dynamics. In this paper, tools from time-scale modeling (TSM) theory [37]-[42] are used to investigate reduced-order dynamics rigorously to help in the stability analysis of the multi-time-scale networked systems. Modeling and synchronizing reduced-order networks of a group of identical agents characterized by continuous singularly-perturbed dynamics over undirected graph topology have been addressed in [43].

The contributions of this paper are as follows:

A) We extend the preliminary findings of [7, 8] to networked formalism of discrete systems with eigen-spectrum gap, thereby exploring the relationship between the graph topology and the coupled system stability framework.

B) We develop a mode-separation methodology of expanding the stabilization control design to the synchronization problem. This is clarified by decomposing the overall network dynamics and designing the controls that synchronize the slow dynamics and the fast ones. By recomposing the slow and fast controllers to the network of two time-scale systems we obtain an approximation of the synchronization behavior imposed for each scale.

C) We established that the controller gain matrices can be determined by solving convex optimization problems in terms of finite linear matrix inequalities with prescribed $\mathbb{H}_{\infty}$ and $\mathbb{H}_{2}$ performance criteria.

Notations: Let $Q^{-1}, Q^{t}$ and $\|Q\|$ denote the inverse, the transpose and induced-matrix-norm of square matrices $Q$, respectively. The notation $Q>0$ is used to represent a symmetric positive-definite matrix $Q$ and $I_{N}$ represents the $N \times N$ identity matrix. If the dimension of any matrix is not not explicitly given, we assume it to have an appropriate dimension for algebraic operations. We use the notation $\bullet$ to denote an element that is induced by symmetry. Sometimes, the arguments of a function will be omitted when no confusion can arise.

\section{Graph theory}

In the sequel, we recall some definitions and properties of Graph theory, which will be used throughout the paper. A weighted graph is a triple $\mathbb{G}=(\mathbb{V}, \mathbb{E}, \mathbb{W})$ consisting of a node (vertex) set $\mathbb{V}=\{1, \cdots n\}$ with cardinality $|\mathbb{V}|=n$, an edge set $\mathbb{E} \subset \mathbb{V} \times \mathbb{V}$ with cardinality $|\mathbb{E}|=m$, a positive weight set $\mathbb{W}$ with cardinality $|\mathbb{W}|=m$, a weighted adjacency matrix $\mathbb{A}=\left[a_{i j}\right]$ with non-negative adjacent elements $a_{i j}$ and the corresponding vector of weights $\mathbf{w}$ with the order $\mathbf{w}_{i j}$ refers to the weight of the edge $\{i, j\}$ [33]. In what follows, we consider undirected graph such that $(i, j) \in \mathbb{E}$ is equivalent to $(j, i) \in \mathbb{E}$. In addition, we consider that the graph $\mathbb{G}$ contains no self-loop $(\forall i=1, \cdots n)$, one has $(i, i) \notin \mathbb{E}$. The adjacency matrix associated with $\mathbb{G}$ is define as $\mathbb{A}=\left[a_{i j}\right] \in \mathfrak{R}^{n \times n}$ such that

$$
\left\{\begin{array}{l}
a_{i j}>0 \quad \text { if }(i, j) \in \mathbb{E} \\
a_{i j}=0 \quad \text { otherwise }
\end{array}\right.
$$

The (graph) Laplacian of $\mathbb{G}$ is a rank deficient and symmetric matrix defined by

$$
\begin{aligned}
\mathbb{L}(\mathbb{G}) & :=\mathbb{E}(\mathbb{G}) \mathbb{E}(\mathbb{G})^{t}=\Delta(\mathbb{G})-\mathbb{A}(\mathbb{G}) \\
& :=\left[\ell_{i j}\right], \ell_{i j}=-a_{i j}, \ell_{i i}=\sum_{j=1}^{n} a_{i j}
\end{aligned}
$$

Based on the definition of $\mathbb{L}$, the any of its rows sum is zero. Moreover, $\mathbb{L}(\mathbb{G})$ ha eigenvalues set $0=\lambda_{1}(\mathbb{G}) \leq \cdots \leq \lambda_{n}(\mathbb{G})=$ $\lambda_{\max }(\mathbb{G})$ and associated with the set eigenvectors $v_{1}:=\frac{1}{n} \mathbf{1}, v_{2}, \ldots, v_{n}$. An attraction of these dynamics is that all subsystems converge to the consensus space defined as $\mathscr{X}_{c}=\left\{\mathbf{y} \in \mathfrak{R}^{n p} \mid \mathbf{y}_{1}(k)=\cdots=\mathbf{y}_{n}(k)\right\}$ when $\mathbb{G}$ is a connected graph [33].

Definition 2.1. In the graph $\mathbb{G}=(\mathbb{V}, \mathbb{E})$, a path of length $p$ is defined as the union of edges as follows:

$$
\bigcup_{m=1}^{p}\left(i_{m}, j_{m}\right) \Rightarrow i_{m+1}=j_{m}, \forall m \in\{1, \cdots p-1\}
$$

The agent $j$ is said to be connected with agent $i$ if there one path exists joining $i$ with $j$, i.e. $i_{1}=i$ and $j_{p}=j$. If every two different agents has at least one path connecting them, the graph is said to be connected. Henceforth, we assume that the undirected graph $\mathbb{G}$ is connected.

The following remark gives some important characteristic of the graph and its Laplacian matrix.

Remark 2.2. Let $\lambda_{1} \leq \lambda_{1} \leq \cdots \leq \lambda_{n}$ be the eigenvalues of $\mathbb{L}$. It follows from [32] that 
- $\lambda_{1}=0$ is a unique zero eigenvalue of $\mathbb{L}$ corresponding to the eigenvector $\mathbb{I} \triangleq[1,1, \cdots, 1]^{t}$.

- $\lambda_{2}$ is strictly positive if and only if the graph $\mathbb{G}$ is connected. This means that $\mathbb{L} \geq 0$.

- $\lambda_{1}=0$ is an eigenvalue with multiplicity $r$ of the matrix $\mathbb{L} \otimes I_{r}$. Moreover, $\mathbb{L} \otimes I_{r}$ has $r$ different normalized eigenvectors given by $\mathbb{I} \otimes e_{i}, i=1, \ldots, r$ where $e_{i} \in \mathfrak{R}^{r}$ is the column vector whose $i^{\text {th }}$ element is 1 and others are zeros.

- An orthonormal matrix exists: $T \in \mathfrak{R}^{n \times n}, \mathbb{T}^{t}=\mathbb{T}^{t} \mathbb{T}=I_{n}$ such that $\mathbb{T} \mathbb{L} \mathbb{T}^{t}=\mathbb{D} \triangleq$ diag $\left(\lambda_{1}, \lambda_{2}, \cdots, \lambda_{n}\right)$

\section{Mode-separation of discrete time dynamical systems}

There are a wide class of linear discrete-time control systems with eigenvalue-separation. By reordering and/or rescaling of states, linear discrete system can be cast into the form

$$
\begin{aligned}
x(k+1) & =A_{1} x(k)+A_{2} z(k)+B_{1} u(k)+\Gamma_{1} \omega(k), \\
z(k+1) & =A_{3} x(k)+A_{4} z(k)+B_{2} u(k)+\Gamma_{2} \omega(k), \\
y(k) & =C_{1} x(k)+C_{2} z(k)
\end{aligned}
$$

where the disturbance weighting matrices are $\Gamma_{1} \in \mathfrak{R}^{n_{1} \times s}, \Gamma_{2} \in \mathfrak{R}^{q \times s}$. We seek to determine the conditions under which the modes of discrete systems can be separated. In (3.1), the state vector is formed by $x(k) \in \mathfrak{R}^{n_{1}}$ and $z(k) \in \mathfrak{R}^{n_{2}}$, and the control is $u(k) \in \mathfrak{R}^{m}$ and the disturbance vector $\omega(k) \in \mathfrak{R}^{s}$.

Assumption 1. Let $n=n_{1}+n_{2}$. System (3.1) is asymptotically Schur stable and its eigen-spectrum

$$
\begin{aligned}
& 1>\left|\lambda_{1}\right|>\cdots>\left|\lambda_{n_{1}}\right|>\left|\lambda_{n_{1}+1}\right|>\cdots>\left|\lambda_{n}\right| \triangleq \lambda\left(A_{s}\right) \cup \lambda\left(A_{f}\right) \\
& \lambda\left(A_{s}\right)=\left\{\lambda_{1}, \cdots, \lambda_{n_{1}}\right\}, \lambda\left(A_{f}\right)=\left\{\lambda_{n_{1}+1}, \cdots, \lambda_{n}\right\}
\end{aligned}
$$

possesses a gap expressed by $\mu \triangleq\left|\lambda_{n_{1}+1}\right| /\left|\lambda_{n_{1}}\right|<<1$

A standard assumption in time-scale modeling theory, which ensures the well-posedness of (3.1) is that following.

Assumption 2. The matrix $A_{4}$ is invertible.

When Assumption 1 is met, then system (3.1) is called a two-time-scale system. To this end $\lambda\left(A_{s}\right), \lambda\left(A_{f}\right)$ define, respectively, the eigenvalues of the slow (dominant) parts and are the eigenvalues of the fast (non-dominant) parts of system (3.1). A useful interpretation of (3.2) is that $\left[A_{f}\right]^{k}$ tends to zero much quicker that $\left[A_{s}\right]^{k}$. Recalling the facts for any square invertible matrix $P$ that

$$
\left|\lambda_{\max }\right| \leq\|P\|, \quad 1 /\left|\lambda_{\min }\right| \leq\left\|P^{-1}\right\|
$$

An alternative expression of the eigen-spectrum property is

$$
\left\|A_{s}^{-1}\right\|\left\|A_{f}\right\|<<1
$$

which designates a matrix norm condition of mode separation in linear discrete systems.

Remark 3.1. By looking at system (3.1) with property (3.2) or (3.3), it is significant that it enjoys the mode-separation implicitly through the recognition of a gap in the eigen-spectrum.

\subsection{Mode separation in networked systems}

We consider a network of $n$ identical linear discrete systems having an eigen-spectrum gap in the manner of (3.2). For any $i=1, \cdots, n$ where the $i^{t h}$ system at discrete instant $k$, represented by the state $\left[x_{i}(k), z_{i}(k)\right] \in \mathfrak{R}^{n_{1}+n_{2}}$ and the input $u(k) \in \mathfrak{R}^{m}$, is given by

$$
\begin{aligned}
x_{i}(k+1) & =A_{1} x_{i}(k)+A_{2} z_{i}(k)+B_{1} u_{i}(k)+\Gamma_{1} \omega_{i}(k), \\
z_{i}(k+1) & =A_{3} x_{i}(k)+A_{4} z_{i}(k)+B_{2} u_{i}(k)+\Gamma_{2} \omega_{i}(k), \\
y_{i}(k) & =C_{1} x_{i}(k)+C_{2} z_{i}(k) \\
A_{1} & \in \Re^{n_{1} \times n_{1}}, A_{2} \in \mathfrak{R}^{n_{1} \times n_{2}}, A_{3} \in \mathfrak{R}^{n_{2} \times n_{1}}, \\
A_{4} & \in \mathfrak{R}^{n_{2} \times n_{2}}, \operatorname{rank}\left(B_{1}\right)=\operatorname{rank}\left(B_{2}\right)=m
\end{aligned}
$$

The consensus problem of $n$ systems is first introduced: 
Definition 3.2. The $n$ discrete systems with mode-separation defined by (3.4) achieve asymptotic synchronization using local information if there exists a state protocol of the form

$$
u_{i}(k)=\mathbb{K}_{1} \sum_{j=1}^{n} a_{i j}\left[x_{i}(k)-x_{j}(k)\right]+\mathbb{K}_{2} \sum_{j=1}^{n} a_{i j}\left[z_{i}(k)-z_{j}(k)\right]
$$

where $\mathbb{K}_{1} \in \Re^{m \times n_{1}}, \mathbb{K}_{2} \in \Re^{m \times n_{2}}$ such that

$$
\lim _{k \rightarrow \infty}\left\|x_{i}(k)-x_{j}(k)\right\|=0, \lim _{k \rightarrow \infty}\left\|z_{i}(k)-z_{j}(k)\right\|=0
$$

The prime objective hereafter is the characterization of the local controllers that use local information and asymptotically synchronize the two time-scale (TTS) discrete systems defined by (3.4). Toward our objective, we express the collective dynamics characterizing the performance of the collective dynamics of $n$ feedback coupled-systems. In terms of

$$
x(k)=\left[x_{1}^{t}(k), \ldots, x_{n}^{t}(k)\right]^{t} \in \Re^{n n_{1}} \text {, and } z(k)=\left[z_{1}^{t}(k), \ldots, z_{n}^{t}(k)\right]^{t} \in \Re^{n n_{2}}
$$

we note that the asymptotic synchronization (3.6) corresponds to

$$
\lim _{k \rightarrow \infty}\left(\mathbb{L} \otimes I_{n_{1}}\right) x(k)=0, \lim _{k \rightarrow \infty}\left(\mathbb{L} \otimes I_{n_{2}}\right) z(k)=0
$$

Invoking the fact that $\mathbb{T L}=\mathbb{D} \mathbb{T}$, it follows that (3.7) can be expressed as

$$
\lim _{k \rightarrow \infty}\left(\mathbb{D} \otimes I_{n_{1}}\right)\left(\mathbb{T} \otimes I_{n_{1}}\right) x(k)=0, \text { and } \lim _{k \rightarrow \infty}\left(\mathbb{D} \otimes I_{n_{2}}\right)\left(\mathbb{T} \otimes I_{n_{2}}\right) z(k)=0
$$

\subsection{Closed-loop representation}

On substituting protocol (3.5) in system (3.4), we obtain the closed-loop dynamics:

$$
\begin{aligned}
& x(k+1)=\hat{\mathbf{A}}_{1} x(k)+\hat{\mathbf{A}}_{2} z(k)+\hat{\Gamma}_{1} \omega(k), \\
& z(k+1)=\hat{\mathbf{A}}_{3} x(k)+\hat{\mathbf{A}}_{4} z(k)+\hat{\Gamma}_{2} \omega(k)
\end{aligned}
$$

where

$$
\begin{aligned}
& \hat{\mathbf{A}}_{1}=\left(I_{n} \otimes A_{1}\right)-\left(I_{n} \otimes B_{1} \mathbb{K}_{1}\right)\left(\mathbb{L} \otimes I_{n_{1}}\right), \\
& \hat{\mathbf{A}}_{2}=\left(I_{n} \otimes A_{2}\right)-\left(I_{n} \otimes B_{1} \mathbb{K}_{2}\right)\left(\mathbb{L} \otimes I_{n_{1}}\right), \\
& \hat{\mathbf{A}}_{3}=\left(I_{n} \otimes A_{3}\right)-\left(I_{n} \otimes B_{2} \mathbb{K}_{1}\right)\left(\mathbb{L} \otimes I_{n_{1}}\right), \\
& \hat{\mathbf{A}}_{4}=\left(I_{n} \otimes A_{4}\right)-\left(I_{n} \otimes B_{2} \mathbb{K}_{2}\right)\left(\mathbb{L} \otimes I_{n_{1}}\right), \\
& \hat{\Gamma}_{1}=\left(I_{n} \otimes \Gamma_{1}\right), \hat{\Gamma}_{2}=\left(I_{n} \otimes \Gamma_{2}\right) .
\end{aligned}
$$

It is significant to notice that unlike the invertibility of matrix $I_{2}-A_{4}$, we can not guarantee that the matrix $I_{2}-\hat{\mathbf{A}}_{4}$ is non-singular. Hence, the well-posedness of the closed-loop dynamics (3.9) has also to be guaranteed by the selection of the matrix gains. We now proceed by making another transformation of variables

$$
\widehat{x}(k)=\left(\mathbb{T} \otimes I_{n_{1}}\right) x(k), \widehat{z}(k)=\left(\mathbb{T} \otimes I_{n_{2}}\right) z(k),
$$

converts the aggregate dynamics (3.9) into the form

$$
\left[\begin{array}{l}
\widehat{x}(k+1) \\
\widehat{z}(k+1)
\end{array}\right]=\left[\begin{array}{ll}
\widehat{\mathbf{A}}_{1} & \widehat{\mathbf{A}}_{2} \\
\widehat{\mathbf{A}}_{3} & \widehat{\mathbf{A}}_{4}
\end{array}\right]\left[\begin{array}{c}
\widehat{x}(k) \\
\widehat{z}(k)
\end{array}\right]+\left[\begin{array}{c}
\widehat{\Gamma}_{1} \\
\widehat{\Gamma}_{2}
\end{array}\right] \omega(k)
$$

where $\widehat{\Gamma}_{1}=\left(\mathbb{T} \otimes I_{n_{1}}\right)\left(I_{n} \otimes \Gamma_{1}\right), \widehat{\Gamma}_{2}=\left(\mathbb{T} \otimes I_{n_{1}}\right)\left(I_{n} \otimes \Gamma_{2}\right)$, and

$$
\begin{aligned}
& \widehat{\mathbf{A}}_{1}=\left(I_{n} \otimes A_{1}\right)-\left(I_{n} \otimes B_{1} \mathbb{K}_{1}\right)\left(\mathbb{D} \otimes I_{n_{1}}\right), \\
& \widehat{\mathbf{A}}_{2}=\left(I_{n} \otimes A_{2}\right)-\left(I_{n} \otimes B_{1} \mathbb{K}_{2}\right)\left(\mathbb{D} \otimes I_{n_{1}}\right), \\
& \widehat{\mathbf{A}}_{3}=\left(I_{n} \otimes A_{3}\right)-\left(I_{n} \otimes B_{2} \mathbb{K}_{1}\right)\left(\mathbb{D} \otimes I_{n_{1}}\right), \\
& \widehat{\mathbf{A}}_{4}=\left(I_{n} \otimes A_{4}\right)-\left(I_{n} \otimes B_{2} \mathbb{K}_{2}\right)\left(\mathbb{D} \otimes I_{n_{1}}\right) .
\end{aligned}
$$

The following results stand out:

Proposition 3.3. The closed-loop system (3.10)-(3.11) can be decoupled in $n$ independent TTS systems. 
Proof: Invoking the properties of Kronecker products [3], one uses the fact that for any matrices $\mathbb{M}, \mathbb{N}$ of appropriate dimensions we have

$$
\begin{aligned}
\left(I_{n} \otimes \mathbb{M}\right)-\left(I_{n} \otimes \mathbb{N}\right)\left(\mathbb{D} \otimes I_{m}\right) & =\left(I_{n} \otimes \mathbb{M}\right)-(\mathbb{D} \otimes \mathbb{N}) \\
& =\operatorname{diag}[\mathbb{M}, \cdots, \mathbb{M}]-\operatorname{diag}\left[\lambda_{1} \mathbb{N}, \cdots, \lambda_{n} \mathbb{N}\right] \\
& =\operatorname{diag}\left[\mathbb{M}-\lambda_{1} \mathbb{N}, \cdots, \mathbb{M}-\lambda_{n} \mathbb{N}\right]
\end{aligned}
$$

which eventually results in

$$
\begin{aligned}
& \widehat{\mathbf{A}}_{1}=\operatorname{diag}\left[A_{1}-\lambda_{1} B_{1} \mathbb{K}_{1}, \cdots, A_{1}-\lambda_{n} B_{1} \mathbb{K}_{1}\right] \\
& \widehat{\mathbf{A}}_{2}=\operatorname{diag}\left[A_{2}-\lambda_{1} B_{1} \mathbb{K}_{2}, \cdots, A_{2}-\lambda_{n} B_{1} \mathbb{K}_{2}\right] \\
& \widehat{\mathbf{A}}_{3}=\operatorname{diag}\left[A_{3}-\lambda_{1} B_{2} \mathbb{K}_{1}, \cdots, A_{3}-\lambda_{n} B_{2} \mathbb{K}_{1}\right] \\
& \widehat{\mathbf{A}}_{4}=\operatorname{diag}\left[A_{4}-\lambda_{1} B_{2} \mathbb{K}_{2}, \cdots, A_{4}-\lambda_{n} B_{2} \mathbb{K}_{2}\right]
\end{aligned}
$$

This in turn casts the closed-loop system (3.10) into the form

$$
\left[\begin{array}{c}
\widehat{x}_{i}(k+1) \\
\widehat{z}_{i}(k+1)
\end{array}\right]\left[\begin{array}{ll}
\left(A_{1}-\lambda_{i} B_{1} \mathbb{K}_{1}\right) & \left(A_{2}-\lambda_{i} B_{1} \mathbb{K}_{2}\right) \\
\left(A_{3}-\lambda_{i} B_{2} \mathbb{K}_{1}\right) & \left(A_{4}-\lambda_{i} B_{2} \mathbb{K}_{2}\right)
\end{array}\right]\left[\begin{array}{c}
\widehat{x}_{i}(k) \\
\widehat{z}_{i}(k)
\end{array}\right]+\left[\begin{array}{c}
\widehat{\Gamma}_{1} \\
\widehat{\Gamma}_{2}
\end{array}\right] \omega(k)
$$

for $i=1, \cdots \cdots, n$, which is the desired result.

Proposition 3.4. The asymptotic synchronization problem under consideration with local state information becomes a problem of feedback simultaneous stabilization of systems in (3.12) for $i=2 ; \cdots ; n$.

Proof: Recall that (3.8) can be cast into

$$
\lim _{k \rightarrow \infty}\left(\mathbb{D} \otimes I_{n_{1}}\right) \widehat{x}=0, \lim _{k \rightarrow \infty}\left(\mathbb{D} \otimes I_{n_{2}}\right)\left(\mathbb{T} \otimes I_{n_{1}}\right) \widehat{z}=0 .
$$

In view of the fact that $\mathbb{D}=\operatorname{diag}\left[\lambda_{1}, \cdots, \lambda_{n}\right], \lambda_{1} \equiv 0$, it follows that the asymptotic synchronization condition reduces to

$$
\lim _{k \rightarrow \infty} \widehat{x}_{i}=0, \lim _{k \rightarrow \infty} \widehat{z}_{i}=0, i=2, \cdots, n
$$

which completes the proof.

Now, it follows from the definition of $\mathbb{T}$, that the following change of variables

$$
\widehat{x}(k)=\left(\mathbb{T}^{t} \otimes I_{n_{1}}\right) x(k), \widehat{z}(k)=\left(\mathbb{T}^{t} \otimes I_{n_{2}}\right) z(k)
$$

also hold.

Proceeding further and following the time-scale design theory [11]-[40] with $\omega_{i}(k) \equiv 0$, the consensus manifold depends on the behavior of $[\widehat{x}(k) ; \widehat{z}(k)]$. Effectively, if the discrete-system

$$
\left[\begin{array}{c}
\widehat{x}_{1}(k+1) \\
\widehat{z}_{1}(k+1)
\end{array}\right]=\left[\begin{array}{ll}
A_{1} & A_{2} \\
A_{3} & A_{4}
\end{array}\right]\left[\begin{array}{c}
\widehat{x}_{1}(k) \\
\widehat{z}_{1}(k)
\end{array}\right]
$$

has a stable equilibrium $\left[\widehat{x}^{*}(k) ; \widehat{z}^{*}(k)\right]$, then the original dynamics (3.1) reaches a finite synchronization asymptotically. If the system (3.13) has unstable equilibrium point then all the systems given in (3.4) achieves consensus on divergent paths.

Finally, the well-posedness of dynamics (3.4) is similar to the system (3.10) which in turn is guaranteed if all systems given in (3.12) are also well-posed. It must be emphasized that for $i=1$, the system is well posed due to the non-singularity of $I_{2}-A_{4}$. The rest of the systems in (3.10) are well-posed if $\mathbb{K}_{2}$ is selected such that $\left(A_{4}-\lambda_{i} B_{2} \mathbb{K}_{2}\right)$ invertible for $i=2, \cdots, n$.

\section{Control design}

In this section, we aim to provide a control design method that gives completely decouple structure of the fast and slow modes that appear in the whole closed-loop system. Following the discrete quasi-steady state concept [7, 40], the fast dynamics associated with the small eigenvalues are crucial only within a short period of time. When that transient period finished, they become negligible and the trajectories behavior the original system can be characterized only by its slow dynamics.

Formally, setting $z_{i}(k+1)=z_{i}(k) \triangleq z_{i s}(k)$ in the dynamics (3.4) is the same as neglecting the effect of the fast dynamics. Under this condition, discrete quasi-steady state is given by

$$
z_{i s}(k)=\left[I_{2}-A_{4}\right]^{-1}\left[A_{3} x_{i s}(k)+B_{2} u_{i s}(k)+\Gamma_{2} \omega(k)\right]
$$


As demonstrated in [11] the slow-mode control law $u_{s}(k)=\mathbb{K}_{1} x_{s}(k)$ and the fast-mode control law $u_{f}(k)=\mathbb{K}_{2} z_{f}(k)$ will eventually produce a composite control law $u_{c}(k)$ based on $u_{c}(k)=u_{s}(k)+u_{f}(k)$.

Note that the gains $\mathbb{K}_{1}, \mathbb{K}_{2}$ can be synthesized for slow and fast modes subject to specified performance objective. To this end and following a discrete-time quasi-steady-state technique [9]-[12], it can be readily investigated, the aggregate model (3.10)-(3.11) can be separated into a slow dynamics

$$
\begin{aligned}
x_{i s}(k+1) & =\left[\widehat{\mathbf{A}}_{o}-\lambda_{i} \widehat{\mathbf{B}}_{o} \mathbb{K}_{1}\right] x_{i s}(k)+\Gamma_{o} \omega(k), \\
y_{i s}(k) & =\left[\widehat{\mathbf{C}}_{o}+\widehat{\mathbf{D}}_{o} \mathbb{K}_{1}\right] x_{i s}(k), \\
z_{i s}(k) & =-\left(I-\widehat{\mathbf{A}}_{4}^{-1}\right)\left[\widehat{\mathbf{A}}_{3}-\lambda_{i} \widehat{\mathbf{B}}_{2} \mathbb{K}_{2}\right] x_{i s}(k)+\Gamma_{2} w(k), \\
\widehat{\mathbf{A}}_{o} & =\widehat{\mathbf{A}}_{1}+\widehat{\mathbf{A}}_{2}\left(I-\widehat{\mathbf{A}}_{4}\right)^{-1} \widehat{\mathbf{A}}_{3} \\
\widehat{\mathbf{B}}_{o} & =\widehat{\mathbf{A}}_{1}+\widehat{\mathbf{A}}_{2}\left(I-\widehat{\mathbf{A}}_{4}\right)^{-1} \widehat{\mathbf{B}}_{2}, \\
\widehat{\mathbf{C}}_{o} & =\widehat{\mathbf{C}}_{1}+\widehat{\mathbf{C}}_{2}\left(I-\widehat{\mathbf{A}}_{4}\right)^{-1} \widehat{\mathbf{A}}_{3}, \\
\widehat{\mathbf{D}}_{o} & =\widehat{\mathbf{C}}_{2}\left(I-\widehat{\mathbf{A}}_{4}\right)^{-1} \widehat{\mathbf{B}}_{2} \\
\Gamma_{o} & =\Gamma_{1}+\widehat{\mathbf{A}}_{2}\left(I-\widehat{\mathbf{A}}_{4}\right)^{-1} \Gamma_{2},
\end{aligned}
$$

of order $n_{1}$, and a fast dynamics:

$$
\begin{aligned}
x_{i f}(k+1) & =\left[\widehat{\mathbf{A}}_{4}-\lambda_{i} \widehat{\mathbf{B}}_{2} \mathbb{K}_{2}\right] x_{i f}(k)+\Gamma_{2} w(k) \\
y_{i f}(k) & =\widehat{\mathbf{C}}_{2} x_{i f}(k)
\end{aligned}
$$

of order $n_{2}$.

We are now in a position to establish the following result:

Theorem 4.1. Let the gain matrices $\mathbb{K}_{1}$ and $\mathbb{K}_{2}$ be designed such that for $i=2, \cdots, n$ the matrices

$$
\left[\widehat{\mathbf{A}}_{o}-\lambda_{i} \widehat{\mathbf{B}}_{o} \mathbb{K}_{1}\right],\left[A_{i 4}-\lambda_{i} B_{2} \mathbb{K}_{2}\right]
$$

are all Schur stable. Then, the composite controllers gain

$$
\mathbb{K}_{c}=\left[I_{m}-\mathbb{K}_{2}\left(I_{n_{2}}-A_{4}\right)^{-1} B_{2}\right] \mathbb{K}_{1}-\mathbb{K}_{2}\left(I_{n_{2}}-A_{4}\right)^{-1} A_{3}
$$

asymptotically synchronize systems (3.4) with local state information.

Proof: Following the results of [9] and selecting the gain matrices $\mathbb{K}_{1}$ and $\mathbb{K}_{2}$ to stabilize the slow and fast subsystems (4.1) and (4.2), respectively for $i=2, \cdots, n$, guarantees that

$$
\begin{aligned}
\widehat{x}_{i}(k) & =\widehat{x}_{i s}(k)+\mathbf{O}(\mu), \\
\widehat{z}_{i}(k) & =\left(I_{n_{2}}-A_{i 4}\right)^{-1}\left[A_{i 3} x_{i s}(k)-\lambda_{i} B_{2} \mathbb{K}_{2} \widehat{x}_{i s}(k)\right]+\widehat{x}_{i f}(k)+\mathbf{O}(\mu)
\end{aligned}
$$

hold for all sufficiently small $\mu>0$ and all $k \in[0, \infty)$. Recalling that the asymptotic synchronization corresponds to

$$
\lim _{k \rightarrow \infty}\left(\mathbb{L} \otimes I_{n_{1}}\right) x(k)=0, \lim _{k \rightarrow \infty}\left(\mathbb{L} \otimes I_{n_{2}}\right) z(k)=0
$$

which holds true in view of

$$
\begin{aligned}
\left(\mathbb{L} \otimes I_{n_{1}}\right) x(k) & =\left(\mathbb{D} \otimes I_{n_{1}}\right) \widehat{x}(k), \\
& =\left[0, \lambda_{2} \widehat{x}_{2}, \cdots, \lambda_{n} \widehat{x}_{n}\right]^{t}, \\
\left(\mathbb{L} \otimes I_{n_{2}}\right) z(k) & =\left(\mathbb{D} \otimes I_{n_{2}}\right) \widehat{z}(k), \\
& =\left[0, \lambda_{2} \widehat{z}_{2}, \cdots, \lambda_{n} \widehat{z}_{n}\right]^{t} .
\end{aligned}
$$

Therefore, the proof is completed.

Remark 4.2. Basically, Theorem 4.1 guarantees asymptotic synchronization of systems (3.1). In order to achieve that, both slow and fast dynamics should be separately synchronized by stabilizing the error between the different dynamics.

Corollary 4.3. Suppose that $\mathbb{K}_{o}$ is designed such that for $i=2, \ldots, n$ that matrices $\left[A_{o}-\lambda_{i} B_{o} \mathbb{K}_{1}\right]$ are Schur stable. If $\left\|A_{4}\right\|_{s}<1$, meaning that the matrix $A_{4}$ has a spectral radius less than 1 , then a lower-order controller with $\mathbb{K}_{1}=\mathbb{K}_{c}$ will asymptotically synchronize systems (3.1) as well. 


\section{5. $\mathbb{H}_{\infty}$ and $\mathbb{H}_{2}$ control design}

We next direct attention to the composite control design problem based on the $\mathbb{H}_{\infty}$ and $\mathbb{H}_{2}$ prescribed performance criteria. Our approach is to pursue a Lyapunov-design implementation of $\mathbb{H}_{\infty}$ and $\mathbb{H}_{2}$ controllers to guarantee stabilizing system (4.1)-(4.2), then we recompose them in the manner of Theorem 4.1. We start with the slow-control design.

\subsection{Slow $\mathbb{H}_{\infty}$ controller}

Consider a Lyapanov candidate function $V_{s}=x_{s}^{t}(k) \mathbb{P}_{s} x_{s}(k), \mathbb{P}_{s}>0$ that is associated with the slow dynamics (4.1). Given a scalar $\gamma_{s}>0$, the objective of slow mode $\mathbb{H}_{\infty}$ control law is to determine the controller $u_{s}(k)=\mathbb{K}_{1} x_{s}(k)$ that leads system (4.1) to stability and guarantees that $\left\|y_{s}(k)\right\|_{2}^{2}<\gamma_{s}^{2}\|\omega(k)\|_{2}^{2}$. The synthesis of the control problem is detailed by the following result:

Theorem 5.1. : The dynamical system (4.1) is stabilized by the control law $u_{s}(k)=\mathbb{K}_{1} x_{s}(k)$ and $\left\|y_{s}(k)\right\|_{2}^{2}<\gamma_{s}^{2}\|\omega(k)\|_{2}^{2}$ if there exist matrices $\mathbb{X}_{s}>0, \mathbb{Y}_{s}$ and a scalar $\gamma_{s}>0$ satisfying the following LMIs for $i=2, \cdots, n$ are feasible

$$
\left[\begin{array}{cccc}
-\mathbb{X}_{s} & 0 & \mathbb{X}_{s} \widehat{\mathbf{A}}_{o}^{t}+\mathbb{Y}_{s}^{t} \widehat{\mathbf{B}}_{o}^{t} & \mathbb{X}_{s} \widehat{\mathbf{C}}_{o}^{t}+\mathbb{Y}_{s}^{t} \widehat{\mathbf{D}}_{o}^{t} \\
\bullet & -\gamma_{s}^{2} \boldsymbol{I} & \Gamma_{o}^{t} & 0 \\
\bullet & \bullet & -\mathbb{X}_{s} & 0 \\
\bullet & \bullet & \bullet & -I
\end{array}\right]<0
$$

The $\mathbb{H}_{\infty}$ slow gain matrix can be obtained as $\mathbb{K}_{1}=\mathbb{Y}_{s} \mathbb{X}_{s}^{-1}$.

Proof: Based on robust control theory [44] that the solution of the slow-mode $\mathbb{H}_{\infty}$ control problem corresponds to obtaining the controller gain $\mathbb{K}_{1}$ that ensures the feasibility of the following inequality:

$$
\Pi_{s}=\Delta V_{s}+y_{s}^{t}(k) y_{s}(k)-\gamma_{s}^{2} \omega^{t}(k) \omega(k)<0 .
$$

Obtaining of difference of the Lyapunov function $\Delta V_{s}$ along the dynamics of (4.1) with the control law $u_{s}(k)=\mathbb{K}_{1} x_{s}(k)$, we rewrite inequality (5.2) in its equivalent form:

$$
\begin{aligned}
\Pi_{s} & =\left[\begin{array}{c}
x_{s}, \\
\omega_{s}
\end{array}\right]^{t} \Xi_{s}\left[\begin{array}{c}
x_{s}, \\
\omega_{s}
\end{array}\right]<0, \\
\Xi_{s} & =\left[\begin{array}{cc}
\Xi s 1 & \Xi_{s 2}, \\
\bullet & -\Xi_{s 3}
\end{array}\right], \\
\Xi_{s} 1 & =-\mathbb{P}_{s}+\left(\widehat{\mathbf{A}}_{o}^{t}+\mathbb{K}_{1}^{t} \widehat{\mathbf{B}}_{o}^{t}\right) \mathbb{P}_{s}\left(\widehat{\mathbf{A}}_{o}+\widehat{\mathbf{B}}_{o} \mathbb{K}_{1}\right)+\left(\widehat{\mathbf{C}}_{o}^{t}+\mathbb{K}_{1}^{t} \widehat{\mathbf{D}}_{o}^{t}\right)\left(\widehat{\mathbf{C}}_{o}+\widehat{\mathbf{D}}_{o} \mathbb{K}_{1}\right), \\
\Xi_{s 2} & =\left(\widehat{\mathbf{A}}_{o}^{t}+\mathbb{K}_{1}^{t} \widehat{\mathbf{B}}_{o}^{t}\right) \mathbb{P}_{s} \Gamma_{o}, \\
\Xi_{s 3} & =\gamma_{s}^{2} I-\Gamma_{o}^{t} \mathbb{P}_{s} \Gamma_{o} .
\end{aligned}
$$

Inequality (5.3) implies that $\Xi_{s}<0$. Employing Schur complements to $\Xi_{s}<0$ and using the following congruent transformation $\mathbb{X}_{s}, I, \mathbb{X}_{s}, I$ with $\mathbb{X}_{s}=\mathbb{P}_{s}^{-1}, \mathbb{K}_{1} \mathbb{X}_{s}=\mathbb{Y}_{s}$, we obtain the LMI (5.1).

\subsection{Fast $\mathbb{H}_{\infty}$-control}

Using the same procedure of the slow-mode case, consider the Lyapunov function $V_{f}=x_{f}^{t}(k) \mathbb{P}_{f} x_{f}(k), \mathbb{P}_{f}>0$ associated with the fast-dynamics (4.2). Given a scalar $\gamma_{f}>0$, the objective of fast-mode $\mathbb{H}_{\infty}$ control law is to obtain the controller $u_{f}(k)=\mathbb{K}_{2} x_{f}(k)$ that stabilizes system (4.2) and guaranteeing that $\left\|y_{f}(k)\right\|_{2}^{2}<\gamma_{f}^{2}\|\omega(k)\|_{2}^{2}$. The synthesis of the control problem is detailed by the following result:

Theorem 5.2. : System (4.2) is stabilizable by the controller $u_{f}(k)=\mathbb{K}_{2} x_{f}(k)$ and $\left\|y_{f}(k)\right\|_{2}^{2}<\gamma_{f}^{2}\|\omega(k)\|_{2}^{2}$ if there exist matrices $\mathbb{X}_{f}>0, \mathbb{Y}_{f}$ and a scalar $\gamma_{f}>0$ such that such that the following LMIs for $i=2, \cdots, n$ are feasible

$$
\left[\begin{array}{cccc}
-\mathbb{X}_{f} & 0 & \mathbb{X}_{f} \widehat{\mathbf{A}}_{4}^{t}+\mathbb{Y}_{f}^{t} \widehat{\mathbf{B}}_{2}^{t} & \mathbb{X}_{f} \widehat{\mathbf{C}}_{2}^{t} \\
\bullet & -\gamma_{f}^{2} I & \Gamma_{2}^{t} & 0 \\
\bullet & \bullet & -\mathbb{X}_{f} & 0 \\
\bullet & \bullet & \bullet & -I
\end{array}\right]<0
$$

The $\mathbb{H}_{\infty}$ fast gain matrix can be determined by $\mathbb{K}_{2}=\mathbb{Y}_{f} \mathbb{X}_{f}^{-1}$.

Proof: The proof is similar to the proof of Theorem 5.1.

We combine the results of Theorems 4.1, 5.1 and 5.2, such that the composite $\mathbb{H}_{\infty}$ control law is obtained by the following result: 
Lemma 5.3. Consider the dynamical system (4.1)-(4.2) and let $\mathbb{X}_{s}>0, \mathbb{Y}_{s}$ and $\mathbb{X}_{f}>0, \mathbb{Y}_{f}$ be the obtained solutions of the LMIs (5.1) and (5.4), respectively. Then, the $\mathbb{H}_{\infty}$ composite controller is obtained in the form

$$
u_{c}(k)=\left[\left(I-\mathbb{Y}_{f} \mathbb{X}_{f}^{-1}\left(I-A_{4}\right)^{-1} B_{2}\right)^{-1} \mathbb{Y}_{s} \mathbb{X}_{s}^{-1}-\mathbb{Y}_{f} \mathbb{X}_{f}^{-1}\left(I-A_{4}\right)^{-1} A_{3}\right] x(k)+\mathbb{Y}_{f} \mathbb{X}_{f}^{-1} z(k)
$$

guarantees that $\|y(k)\|_{2}^{2}<\gamma^{2}\|\omega(k)\|_{2}^{2}$ with $\gamma \in\left[\gamma_{s}, \gamma_{f}\right]$. In addition, it yields an approximation of first-order to the states of the original dynamics (3.4).

If the fast-mode dynamics is asymptotically stable, we can derive a reduced-order $\mathscr{H}_{\infty}$ control based on the following lemma:

Lemma 5.4. Consider the dynamics in (3.1) and assume $X_{s}>0, Y_{s}$ are the solutions obtained by solving LMI (5.1). Then the reduced-order $\mathbb{H}_{\infty}$ control law is given as follows

$$
u_{c}(k)=\mathbb{Y}_{s} \mathbb{X}_{s}^{-1} x(k)
$$

guarantees that $\|y(k)\|_{2}^{2}<\gamma^{2}\|\omega(k)\|_{2}^{2}$ with $\gamma \in\left[\gamma_{s}, \gamma_{f}\right]$. In addition, it yields an approximation of first-order to the states of the original dynamics (3.4).

Proof: The proof follows parallel details to the results in [7, 40].

Remark 5.5. It is worth noting that that the results of Theorems 5.1 and 5.2 and Lemmas 5.3-5.4 are new in the field of two time-scale discrete-time dynamical systems. Morover, it also strengthen the idea that system (3.1) is represent a good model of discrete-time practical engineering dynamics with implicit representation of the mode-separation property.

\section{6. $\mathbb{H}_{2}$ Control design}

Similarly, instead of synthesizing a full-order $\mathbb{H}_{2}$ control, we decompose it into separate $\mathbb{H}_{2}$ controllers for slow and fast modes. Moreover, we recompose the controllers similar ro the manner of Theorem 4.1.

\subsection{Slow $\mathbb{H}_{2}$ controller}

Consider a candidate Lyapunov function $V_{s}=x_{s}^{t}(k) \mathbb{P}_{s 2} x_{s}(k), \mathbb{P}_{s 2}>0$ corresponding the slow dynamics (4.1). The objective of slow-mode $\mathbb{H}_{2}$ control law is to guarantee the stability of closed-loop slow mode and to maintain the $\mathbb{H}_{2}$-objective of the closed loop transfer function $H_{y_{s} w}(s)$ from the exogenous input $\omega$ to controlled output $y_{s}$ as small as possible. Substituting the slow-mode control law $u_{s}(k)=\mathbb{K}_{s 1} x_{s}(k)$ into (4.1), the closed-loop slow subsystem becomes

$$
\begin{aligned}
x_{s}(k+1) & =\widehat{\mathbf{A}}_{c o} x_{s}(k)+\Gamma_{o} \omega(k), \\
y_{s}(k) & =\widehat{\mathbf{C}}_{c o} x_{s}(k), \\
\widehat{\mathbf{A}}_{c o} & =\widehat{\mathbf{A}}_{o}+\widehat{\mathbf{B}}_{o} \mathbb{K}_{s 1}, \widehat{\mathbf{C}}_{c o}=\widehat{\mathbf{C}}_{o}+\widehat{\mathbf{D}}_{o} \mathbb{K}_{s 1} .
\end{aligned}
$$

Based on Lyapunov theory, given the control gain matrix $\mathbb{K}_{s 1}$, the closed-loop dynamics (6.1) become asymptotically stable $\omega(k) \equiv 0$ if

$$
\mathbb{P}_{s 2}-\widehat{\mathbf{A}}_{c o}^{t} \mathbb{P}_{s 2} \widehat{\mathbf{A}}_{c o}>0
$$

Then, we can express the square of the $\mathbb{H}_{2}$-norm of the transfer function $H_{z w}(s)$ in terms of the solution of a Lyapunov equation (controllability Grammian). In addition, its minimization problem with respect to the gain matrix $\mathbb{K}_{s 1}$ is characterized as

$$
\min \left\{\operatorname{Tr}\left[\widehat{\mathbf{C}}_{c o} \mathbb{P}_{s 2} \widehat{\mathbf{C}}_{c o}^{t}\right]: \mathbb{P}_{s 2}-\widehat{\mathbf{A}}_{c o}^{t} \mathbb{P}_{s 2} \widehat{\mathbf{A}}_{c o}+\Gamma_{o} \Gamma_{o}^{t}=0\right\}
$$

where $\operatorname{Tr} r[$.$] represents the trace of a matrix. Since \mathbb{P}_{s 2}<\mathscr{P}$ for any $\mathscr{P}$ satisfying

$$
\mathscr{P}-\widehat{\mathbf{A}}_{c o}^{t} \mathscr{P} \widehat{\mathbf{A}}_{c o}+\Gamma_{o} \Gamma_{o}^{t}<0
$$

it is readily verified that $\left\|H_{z w}(s)\right\|_{2}^{2}=\operatorname{Tr}\left[\widehat{\mathbf{C}}_{c o} \mathbb{P}_{s 2} \widehat{\mathbf{C}}_{c o}^{t}\right]<v$ if and only if there exists $\mathscr{P}>0$ satisfying $(6.2)$ and $\mathbf{T r}\left[\widehat{\mathbf{C}}_{c o} \mathbb{P} s 2 \widehat{\mathbf{C}}_{c o}^{t}\right]<$ $v$. We introduce a new dummy variable $\mathscr{Z}$, to obtian the following synthesis result:

Theorem 6.1. : The dynamical system (4.1) can bw stabilizable by the control law $u_{s}(k)=\mathbb{K}_{s 1} x_{s}(k)$ and $\left\|H_{z w}(s)\right\|_{2}^{2}<v$ for a prescribed $v$ if and only if there exist positive definite matrices $\mathscr{P}, \mathscr{Z}$, and a matrix $\mathscr{Q}$ with appropriate dimensions satisfying the following conditions:

$$
\begin{aligned}
& \operatorname{Tr}(\mathscr{Z})<v,\left[\begin{array}{cc}
\mathscr{Z} & \widehat{\mathbf{C}}_{o} \mathscr{P}+\widehat{\mathbf{D}}_{o} \mathscr{Q} \\
\bullet & \mathscr{P}
\end{array}\right]>0 \\
& {\left[\begin{array}{ccc}
\mathscr{P} & \widehat{\mathbf{A}}_{o} \mathscr{P}+\widehat{\mathbf{B}}_{o} \mathscr{Q} & \Gamma_{o} \\
\bullet & \mathscr{P} & 0 \\
\bullet & \bullet & I
\end{array}\right]>0}
\end{aligned}
$$

Moreover, the slow-mode gain matrix is obtained by $\mathbb{K}_{s 1}=\mathscr{Q} \mathscr{P}-1$

proof: It can be proved based on standard convex analysis similar to procedure presented in [45]. 


\subsection{Fast $\mathbb{H}_{2}$ controller}

Similarly, consider a Lyapunov function $V_{f}=x_{f}^{t}(k) \mathbb{P}_{f 2} x_{f}(k), \mathbb{P}_{f 2}>0$ associated with the fast dynamics (4.2). The objective of fast-mode $\mathbb{H}_{2}$ control law is to guarantee the stability of closed-loop fast-mode and to maintain a prescribed $\mathbb{H}_{2}$-performance the closed loop transfer function $H_{y_{f} w}(s)$ from $\omega$ to $y_{f}$ as small as possible. The corresponding synthesis result is provided by the following result which follows a parallel development to Theorem 6.1:

Theorem 6.2. : System (4.2) is stabilizable by the controller $u_{f}(k)=\mathbb{K}_{f 2} x_{f}(k)$ and $\left\|H_{y_{f} w}(s)\right\|_{2}^{2}<v$ for a prescribed $v$ if and only if there exist matrices $\mathscr{R}>0, \mathscr{S}, \mathscr{W}>0$ such that

$$
\begin{aligned}
& \operatorname{Tr}(\mathscr{W})<v,\left[\begin{array}{cc}
\mathscr{W} & \widehat{\mathbf{C}}_{2} \mathscr{R} \\
\bullet & \mathscr{R}
\end{array}\right]>0, \\
& {\left[\begin{array}{ccc}
\mathscr{R} & \widehat{\mathbf{A}}_{4} \mathscr{R}+\widehat{\mathbf{B}}_{2} \mathscr{S} & \Gamma_{2} \\
\bullet & \mathscr{R} & 0 \\
\bullet & \bullet & I
\end{array}\right]>0}
\end{aligned}
$$

Moreover, the fast gain is given by $\mathbb{K}_{f 2}=\mathscr{S} \mathscr{R}^{-1}$

Once again, by combining Theorems $4.1,6.1$ and 6.2, the composite $\mathbb{H}_{2}$ controller is obtained by the following result:

Lemma 6.3. Consider the dynamical system (3.1). Let $\mathscr{P}>0, \mathscr{Q}, \mathscr{Z}>0$ and $\mathscr{R}>0, \mathscr{S}, \mathscr{W}>0$ be the given solutions of the conditions in (6.3) and (6.4). Then we obtain the $\mathscr{H}_{2}$ composite control as

$$
u_{c}(k)=\left[\left(I-\mathscr{S} \mathscr{R}^{-1}\left(I-A_{4}\right)^{-1} B_{2}\right)^{-1} \mathscr{Q} \mathscr{P}^{-1}-\mathscr{S} \mathscr{R}^{-1}\left(I-A_{4}\right)^{-1} A_{3}\right] x(k)+\mathscr{S} \mathscr{R}^{-1} z(k)
$$

that guarantees the stability of closed-loop system and maintaining the $\mathbb{H}_{2}$-norm of the closed loop transfer function $H_{y w}(s)$ from $\omega$ to $y_{s}$ as small as possible. In addition, it yields an approximation of first-order to the states of the original dynamics (3.1).

Remark 6.4. In a similar way, the results of Theorems 6.1 and 6.2 and Lemmas 6.3 and 5.4 are contributions to the field of discrete systems with mode-separation. It is important to assert the relevance of the permutation and/or scaling in casting the discrete dynamics of the type (3.1) in the structure of two-time-scale discrete modes with the property of implicit characterization of the mode-separation.

\section{Simulation example}

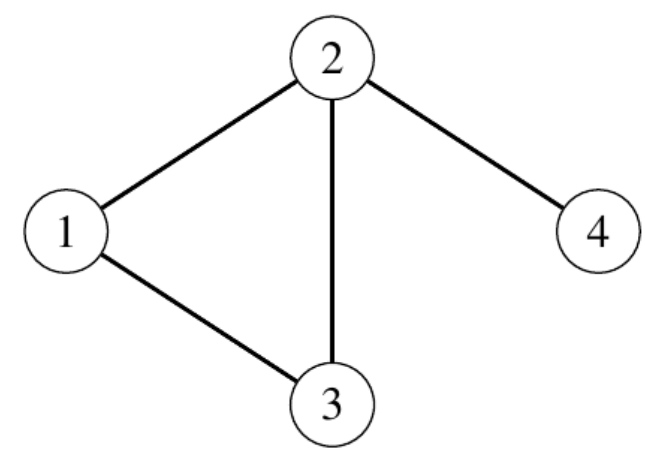

Figure 7.1: Connected topology of 4 agents.

Now, we apply the provided theoretical results to an engine model with dynamometer test. A linearizion is used to obtain the linear model as developed in [46].The state variables are selected as follows: the speed of the rotor, shaft-torque, speed of the engine and amplifier's current.The throttle-servo voltage and dynamometer current are input variables.Consider a group of 4 agent whose graph topology is shown in Fig 7.1. It can be easily shown that the model has a mode separation with two time scales: slow states $\left(n_{1}=2\right)$ and three fast states $\left(n_{2}=3\right)$. Using the data given in [46], the slow dynamics (4.1) is described by

$$
\begin{aligned}
& A_{o}=\left[\begin{array}{cc}
0.762 & 0 \\
-0.029 & 0.689
\end{array}\right], B_{o}=\left[\begin{array}{cc}
0 & 1.049 \\
0.090 & -0.018
\end{array}\right] \\
& C_{o}=\left[\begin{array}{cc}
0 & 1 \\
-0.221 & 8.191
\end{array}\right], D_{o}=\left[\begin{array}{cc}
0 & 0 \\
0.765 & -0.144
\end{array}\right]
\end{aligned}
$$


whereas the fast model (4.2) is given by

$$
A_{4}=\left[\begin{array}{ccc}
0.160 & -0.002 & -0.258 \\
0 & -0.038 & 0 \\
0.231 & 0 & -0.381
\end{array}\right], B_{2}=\left[\begin{array}{cc}
0.702 & -0.083 \\
0 & 22.400 \\
0.142 & 0.026
\end{array}\right], C_{2}=\left[\begin{array}{lll}
0 & 0 & 1
\end{array}\right]
$$

Based on Theorems 5.1 and 5.2, we obtain the $\mathbb{H}_{\infty}$ slow and fast matrix gains as follows

$$
\begin{aligned}
& \mathbb{K}_{1}=\left[\begin{array}{cc}
0.008 & -0.094 \\
0.007 & 0.089
\end{array}\right], \gamma_{s}=0.453 \\
& \mathbb{K}_{2}=\left[\begin{array}{lll}
-0.286 & -0.001 & -0.079 \\
-0.277 & -0.011 & -0.084
\end{array}\right], \gamma_{f}=0.629
\end{aligned}
$$

This gives the $\mathbb{H}_{\infty}$ composite control law as

$$
\begin{aligned}
\mathbb{K}_{c} & =\left[\begin{array}{lllll}
0.054 & 0.030 & -0.288 & 0.012 & -0.078 \\
0.051 & 0.114 & -0.269 & 0.078 & -0.103
\end{array}\right], \\
\gamma_{c} & \in[0.453,0.629] .
\end{aligned}
$$

In addition, applying Theorems 6.1 and 6.2 with $v=1.245$ gives the following $\mathbb{H}_{2}$ slow and fast matrix gains as

$$
\mathbb{K}_{1}=\left[\begin{array}{cc}
0.016 & -0.085 \\
0.002 & 0.097
\end{array}\right], \mathbb{K}_{2}=\left[\begin{array}{lll}
-0.305 & -0.013 & -0.044 \\
-0.225 & -0.001 & -0.103
\end{array}\right]
$$

Based on the gain matrices $\mathbb{K}_{1}$ and $\mathbb{K}_{1}$, the $\mathbb{H}_{2}$ composite control law is obtained:

$$
\mathbb{K}_{c}=\left[\begin{array}{ccccc}
0.0784 & -0.248 & -50.87 & -0.0065 & -0.0771 \\
0.095 & 0.0534 & -144.7 & -0.0047 & -0.246
\end{array}\right]
$$

According to Lemmas 5.3-6.3, the composite gains guarantee good approximation to the closed-loop state trajectories. Figure 7.2 shows the output response of the original system. The output disagreement of all agents are demonstrated in Figs 7.3, 7.4 and 7.5 .

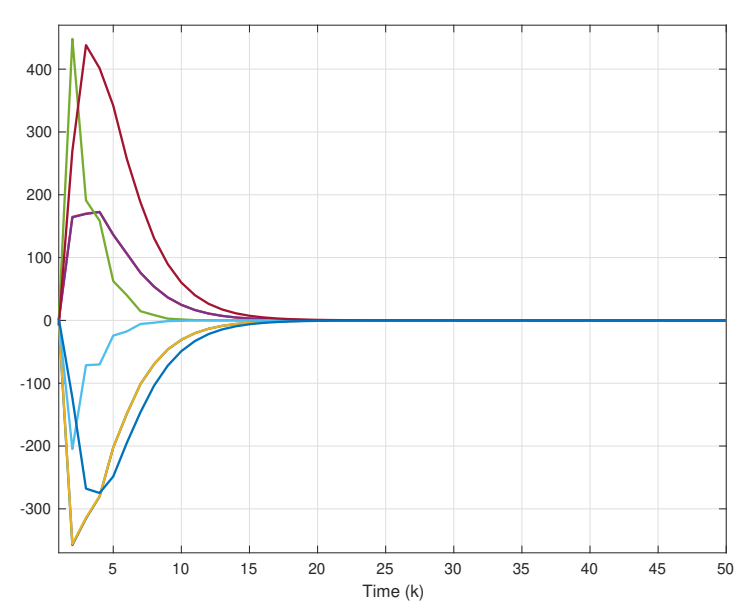

Figure 7.2: Output response of the original system. 


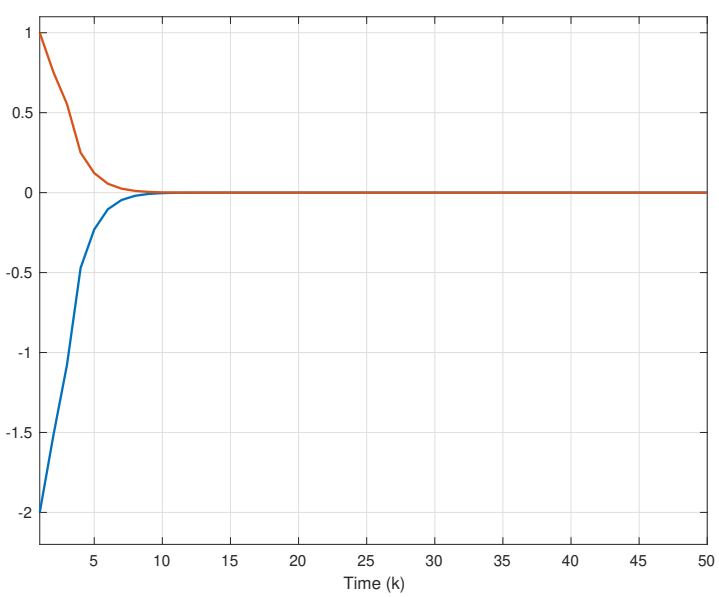

Figure 7.3: Disagreement among outputs $y_{1}(k)-y_{2}(k)$

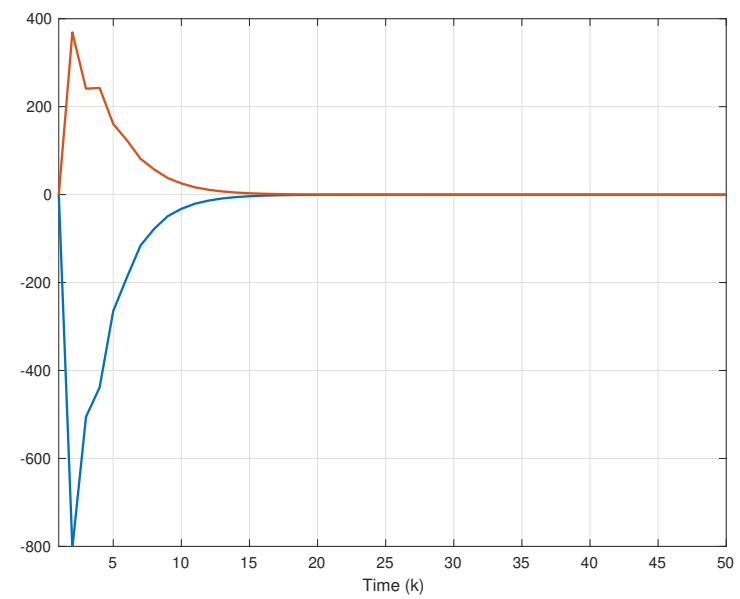

Figure 7.4: Disagreement among outputs $y_{2}(k)-y_{3}(k)$

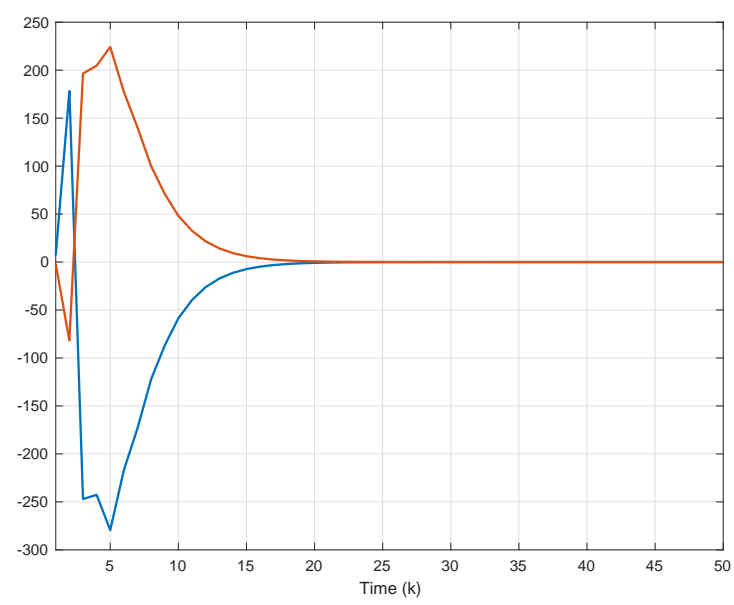

Figure 7.5: Disagreement among outputs $y_{3}(k)-y_{4}(k)$

\section{Conclusions}

This article investigated feedback control synthesis problem of a broad range of discrete-time dynamics that possesses eigenspectrum gap. The fast and slow modes are assumed to be observable and controllable. This assumption constitutes a very mild 
condition and less conservative than assuming observability and controllability of the original dynamical system. Adopting either the $\mathbb{H}_{\infty}$ or $\mathbb{H}_{\infty}$ optimization schemes, we have investigated two-stage design approach based on separate gain matrices for the slow and fast modes. We have constructed a composite controller to obtain first-order approximations to the behavior of the discrete-time dynamics. Moreover, the paper investigates the interactions between multiple time-scale-networked dynamics and gives guarantees on the stability of the disagreement among coupled systems. The addressed effectiveness of the presented methodologies have been demonstrated using a typical application model.

\section{References}

[1] M. S. Mahmoud, M. G. Singh, Large Scale Systems Modelling, Pergamon Press, London, 1981.

[2] P. Kokotovic, H. Khalil, J. O'reilly, Singular perturbation methods in control: analysis and design, Society for Industrial and Applied Mathematics, 1999.

[3] M. S. Mahmoud, M. G. Singh, Discrete Systems: Analysis, Control, and Optimization, Springer-Verlag, Berlin, Germany, 1984.

[4] H. Khalil, F. Chen, $\mathscr{H}_{\infty}$-control of two-time-scale systems, Systems Control Lett., 19, (1992), 35-42.

[5] J. Vian, M. Sawan, $\mathscr{H}_{\infty}$-control for a singularly perturbed aircraft model, Optimal Control Appl. Methods, 15, (1994), 277-289.

[6] E. Fridman, Robust sampled-data $\mathscr{H}_{\infty}$ control of linear singularly perturbed systems, IEEE Trans. Automat. Control, 51(3), (2006), 470-475.

[7] M S. Mahmoud, Order reduction and control of discrete systems, Proc. IEE Part D, 129(4), (1982), 129-135.

[8] M S. Mahmoud, Multi-time scale analysis in discrete systems, J. Eng. Appl. Sci., 2(4), (1983), 301-315.

[9] M. S. Mahmoud, Y. Chen, M. G. Singh, On the eigenvalue assignment in discrete systems with slow and fast modes, Internat. J. Systems Sci., 16(1), (1985), 168-187.

[10] M. S. Mahmoud, Design of observer-based controllers for a class of discrete systems, Automatica, 18(3), (1982), 323-328.

[11] M. S. Mahmoud, Y. Chen, Design of feedback controllers by two-stage methods, Appl. Math. Model., 7(3), (1983), 163-168.

[12] H. A. Othman, N. M. Khraishi, Magdi S. Mahmoud, Discrete regulators with time-scale separation, IEEE Trans. Automat. Control, 30(6), (1985), 293-297.

[13] M. S. Mahmoud, M. G. Singh, On the use of reduced-order Models in output feedback design of discrete systems, Automatica, 21(4), (1985), 485-489.

[14] Y. Cao, W. Yu, W. Ren, G. Chen, An overview of recent progress in the study of distributed multi-agent coordination, IEEE Trans. Ind. Inf., 9(1), (2013),

[15] H. Su, G. Jia, M.Z.Q. Chen, Semi-global containment control of multi-agent systems with intermittent input saturation, J. Frank1. Inst., 8(18), (2015), 3504-3525.

[16] X. Wang, H. Su, Self-triggered leader-following consensus of multi-agent systems with input time delay, Neurocomputing, 330, (2019), 70-77.

[17] H. Su, H. Wu, X. Chen, Observer-based discrete-time nonnegative edge synchronization of networked systems, IEEE Trans. Neural Netw. Learn. Syst., 28(10), (2017), 2446-2455.

[18] J. Zhang, H. Su, Time-varying formation for linear multi-agent systems based on sampled data with multiple leaders, Neurocomputing, 339, (2019),

[19] H. Su, H. Wu, J. Lam, Positive edge-consensus for nodal networks via output feedback, IEEE Trans. Automat. Control, 64(3), (2019), 1244-1249.

[20] W. Ren, R. Bresad, E. Atkins, Information consensus in multivehicle cooperative control: collective group behavior through local interaction, IEEE Control Syst. Mag., 27(2), (2007), 71-82.

[21] H. Su, Y. Sun, Z. Zeng, Semi-global observer-based nonnegative edge consensus of networked systems with actuator saturation, IEEE Trans. Cybern, 50(6) (2020), 2827-2836.

[22] P. Liu, Z. Zeng, J. Wang, Multiple Mittag-Leffler stability of fractional-order recurrent neural networks, IEEE Trans. Syst. Man Cybern. Syst., 47(8), (2017), 2279-2288.

[23] H. Su, H. Wu, X. Chen, M.Z.Q. Chen, Positive edge consensus of complex networks,IEEE Trans. Syst. Man Cybern. Syst., 48(12), (2018), 2242-2250.

[24] P. Liu, Z. Zeng, J. Wang, Multistability analysis of a general class of recurrent neural networks with non-monotonic activation functions and time-varying delays, Neural Netw., 79, (2016), 117-127.

[25] X. Wang, H. Su, Consensus of hybrid multi-agent systems by event-triggered/self-triggered strategy, Appl. Math. Comput., 359, (2019), 490-501.

[26] H. Su, Y. Ye, X. Chen, H. He, Necessary and sufficient conditions for consensus in fractional-order multiagent systems via sampled data over directed graph, IEEE Trans. Syst. Man Cybern. Syst., (2019) Doi: 10.1109/TSMC.2019.2915653, Early Access.

[27] B. J. Karaki, M. S. Mahmoud, Consensus of time-delay stochastic multiagent systems with impulsive behavior and exogenous disturbances, Neurocomputing, 439, (2021), 86-95.

[28] B. J. Karaki, M.S. Mahmoud, Quantised scaled consensus of linear multiagent systems on faulty networks,Internat. J. Systems Sci., (2021), 1-15.

[29] M. S. Mahmoud, B. J. Karaki, Output-Synchronization of Discrete-Time Multiagent Systems: A Cooperative Event-Triggered Dissipative Approach, IEEE Trans. Network Sci. Eng., (2020), Doi: 10.1109/TNSE.2020.3029078, Early Access, .

[30] H. Wu, H. Su, Discrete-time positive edge-consensus for undirected and directed nodal networks, IEEE Trans. Circuits Syst.-II:Exp. Briefs, 65(2), (2018), 221-225.

[31] M. S. Mahmoud, Discrete-time networked control systems, Proc. the Fourth Int. Conference on Mathematical Methods \& Computational Techniques in Science \& Engineering (MMCTSE 2020), London, UK, Paper 103, 2020.

[32] C. Godsil, G. Royle, Algebraic Graph Theory, Springer, 2001.

[33] M. Mesbahi, M. Egerstedt, Graph Theoretic Methods in Multiagent Networks, Princeton Univ. Press, USA, 2010.

[34] M. S. Mahmoud, Stabilization of discrete systems with multiple time scales, IEEE Trans. Automat. Control, 31(2), (1986), 159-162.

[35] D. S. Naidu, A. J. Calise, Singular perturbations and time scales in guidance and control of aerospace systems-A survey, J. Guid. Control Dyn., 24, (2001), 1057-1078.

[36] J. B. Rejeb, I.C. Morarescu, J. Daafouz, Synchronization in networks of linear singularly perturbed systems, Proc. Amer. Control Conf., (2016), 4293-4298.

[37] D. S. Naidu, Singular Perturbation Methodology in Control Systems, Peter Peregrinus Limited, Stevenage Herts, UK, 1988

[38] T. H. S. Li, J. S. Chiou, F. C. Kung, Stability bounds of singularly perturbed discrete systems, IEEE Trans. Automat. Control, 44(10), (1999), 1934-1938.

[39] W. S. Kafri, A. E. Abed, Stability analysis of discrete-time singularly perturbed systems, IEEE Trans. Circuits Systems I Fund. Theory Appl., 43(10), (1996), 848-850.

[40] M. S. Mahmoud, Stabilization of discrete systems with multiple time scales, IEEE Trans. Automat. Control, 31(2), (1986), $159-162$.

[41] D. S. Naidu, A. J. Calise, Singular perturbations and time scales in guidance and control of aerospace systems-A survey, J. Guid. Control Dyn., 24, (2001), 1057-1078.

[42] H. Kando, T. Iwazumi, Multirate digital control design of an optimal regulator via singular perturbation theory, Internat. J. Control, 44, (1986), $1555-1578$.

[43] J. B. Rejeb, I.C. Morarescu, J. Daafouz, Synchronization in networks of linear singularly perturbed systems, Proc. Amer. Control Conf., (2016), 4293-4298.

[44] K. Zhou, J. C. Doyle, Essentials of Robust Control, Prentice-Hall, New Jersey, 1998.

[45] M. C. De Oliveira, J. C. Geromel, J. Bernussou, Extended $\mathscr{H}_{2}$ and $\mathscr{H}_{\infty}$-norm characterizations and controller parametrizations for discrete-time systems, Internat. J. Control, 75(9), (2002), 666-679.

[46] N. Munro, S. M. Hirbad, Multivariable control of an engine/dynamometer test rig, Proc. Seventh IFAC Congress, Helsinki, (1978), $369-376$. 